\title{
EXACTNESS OF A RANK ONE QUANTUM INDUCTION FUNCTOR
}

\author{
JENS G. JENSEN
}

\begin{abstract}
We give a short and elementary proof of the exactness of the induction functor $H_{A}^{0}\left(U_{A} / U_{A}^{0},-\right)$ for $U_{q}\left(\mathfrak{s l}_{2}\right)$.
\end{abstract}

\section{Introduction}

Let $U$ be the quantized universal enveloping algebra (quantum group) associated to a simple finite dimensional Lie algebra $\mathfrak{g}$. Then $U$ has a PoincareBirkhoff-Witt type decomposition $U=U^{-} U^{0} U^{+}$. We may use a given module for the subalgebra $U^{0}$ to construct modules for $U$ by "induction"; in this paper we study such a functor in the case $\mathfrak{g}=\mathfrak{s t}_{2}$. In [1] induction is studied for a quantum algebra over a certain localization of $A=\mathrm{Z}\left[q, q^{-1}\right]$, in particular, exactness is proved in $[1,2.11]$. The proof involves (among other things) specialization to the case $q=1$ and Kempf's vanishing theorem. It is also possible via other specializations to avoid this localization but the complete proof becomes quite long and non-trivial (an alternative proof may be given using Lusztig's canonical bases, see the related results on the quantum coordinate algebra in $[3,29.5]$.)

In this paper we give a short and elementary proof of the exactness of induction in the case $\mathfrak{g}=\mathfrak{s l}_{2}$ where $U$ is an $A$-algebra (no localization). The result in this case is mentioned in $[4,2.3]$ but the proof sketched there is incorrect.

This question was put to me by Henning Haahr Andersen at the University of Aarhus, and it is my pleasure to acknowledge his support and guidance. I am also grateful for his suggestions in relation to the preparation of this paper.

Received November 1, 1996. 


\section{Notation}

Let $A=\mathbf{Z}\left[q, q^{-1}\right], q$ an indeterminate, and let $U$ be the quantized universal enveloping algebra of type $\mathfrak{s l}_{2}$, i.e., $U$ is the $\mathrm{Q}(q)$-algebra generated by $E, F, K, K^{-1}$ with relations

$$
\begin{gathered}
K K^{-1}=1=K^{-1} K, \\
K E K^{-1}=q^{2} E, \quad K F K^{-1}=q^{-2} F, \\
{[E, F]=\frac{K-K^{-1}}{q-q^{-1}} .}
\end{gathered}
$$

Define for $c \in \mathbf{Z}, \quad[c]=\frac{q^{c}-q^{-c}}{q-q^{-1}}, \quad$ and $\quad$ for $\quad t \in \mathbf{N}, \quad[t] !=\prod_{j=1}^{t}[j] \quad$ and $\left[\begin{array}{l}c \\ t\end{array}\right]=\prod_{j=1}^{t} \frac{q^{c-j+1}-q^{-c+j-1}}{q^{j}-q^{-j}}$. In particular, $\left[\begin{array}{l}c \\ 0\end{array}\right]=1$ and $[0] !=1$, and $\left[\begin{array}{l}c \\ t\end{array}\right]=0$ for $t>c \geq 0$. For all $c, t$ as above, the $\left[\begin{array}{l}c \\ t\end{array}\right]$ belong to $A$. We define $E^{(r)}=\frac{1}{[r] !} E^{r}, F^{(r)}=\frac{1}{[r] !} F^{r}$; let $U_{A}$ be the $A$-subalgebra of $U$ generated by $E^{(r)}, F^{(r)}, K, K^{-1},(r=0,1, \ldots)$. We have a decomposition

$$
U_{A}=U_{A}^{-} U_{A}^{0} U_{A}^{+}
$$

[2, Thm. 6.7] where $U_{A}^{-}$is generated by the $F^{(r)}, U_{A}^{+}$by the $E^{(r)}$, and $U_{A}^{0}$ by $K, K^{-1},\left[\begin{array}{c}K ; c \\ t\end{array}\right]$.

Define for $c \in \mathbf{Z}, t \in \mathbf{N}$

$$
\left[\begin{array}{c}
K ; c \\
t
\end{array}\right]=\prod_{j=1}^{t} \frac{K q^{c-j+1}-K^{-1} q^{-c+j-1}}{q^{j}-q^{-j}}
$$

these elements belong to $U_{A}^{0}$.

For $m \in \mathbf{Z}$ we define a character $\chi_{m}: U_{A}^{0} \rightarrow A(\mathrm{cf}$. [1], Lemma 1.1) by

$$
\chi_{m}\left(K^{ \pm}\right)=q^{ \pm m}, \chi_{m}\left(\left[\begin{array}{c}
K ; c \\
t
\end{array}\right]\right)=\left[\begin{array}{c}
m+c \\
t
\end{array}\right], c \in \mathbf{Z}, t \in \mathbf{N}
$$

and for a $U_{A}^{0}$-module $M$ the $m$ 'th weight space (of type 1, cf. [1, 1.2])

$$
M_{m}=\left\{v \in M \mid \forall u \in U_{A}^{0}: u v=\chi_{m}(u) v\right\}
$$

We may consider $A$ as a $U_{A}^{0}$-module by letting $u \in U_{A}^{0}$ act as multiplication by $\chi_{m}(u)$; this $U_{A}^{0}$-module is (by abuse of notation) written simply as $\chi_{m}$.

Let $M$ be a $U_{A}$-module, and define

$$
\mathscr{F}(M)=\left\{v \in \bigoplus_{\nu} M_{\nu} \mid E^{(r)} v=0=F^{(r)} v \text { for } r \gg 0\right\}
$$

$\mathscr{F} M$ is a submodule of $M$ (cf. the proof of Lemma 3 below) and we say that $M$ is integrable if $\mathscr{F} M=M$. Let $\mathscr{U}_{A}$ be the category of $U_{A}$-modules and let $\mathscr{C}_{A}$ be the full subcategory of $\mathscr{U}_{A}$ whose objects are the integrable $U_{A}$-mod- 
ules; then $\mathscr{F}$ is a functor $\mathscr{U}_{A} \rightarrow \mathscr{C}_{A}$. We let $\mathscr{C}_{A}^{\prime}$ denote the category of "integrable" $U_{A}^{0}$-modules (meaning that they are direct sums of their weight spaces.)

We define an induction functor as in [1, 1.9-10],

$$
H^{0}\left(U_{A} / U_{A}^{0},-\right)=\mathscr{F} \circ \operatorname{Hom}_{U_{A}^{\prime}}\left(U_{A},-\right): \mathscr{C}_{A}^{\prime} \rightarrow \mathscr{C}_{A}
$$

where, if $M$ is a $U_{A}^{0}$-module, $U_{A}$ acts on $\operatorname{Hom}_{U_{A}^{0}}\left(U_{A}, M\right)$ as follows:

$$
(u f)(x)=f(x u), x, u \in U_{A}, f \in \operatorname{Hom}_{U_{A}^{0}}\left(U_{A}, M\right)
$$

\section{Exactness of the induction functor}

Proposition 1. Let $m \in Z$ Z. If $m<0$ then $H_{A}^{0}\left(\chi_{m}\right)=0$. If $m \geq 0$ then $H_{A}^{0}\left(\chi_{m}\right)$ is a free A-module; it has a basis $e_{0}, e_{1}, \ldots, e_{m}$ such that for all $r \geq 0$ and all $i \in\{0, \ldots, m\}$ we have

$$
\begin{aligned}
e_{i} & \in H_{A}^{0}\left(\chi_{m}\right)_{m-2 i} \\
E^{(r)} e_{i} & =\left[\begin{array}{c}
i \\
r
\end{array}\right] e_{i-r}, i=0, \ldots, m \\
F^{(r)} e_{i} & =\left[\begin{array}{c}
m-i \\
r
\end{array}\right] e_{i+r}, i=0, \ldots, m
\end{aligned}
$$

where we set $e_{s}=0$ for $s<0$ or $s>m$.

Proof. Same as [1, Proposition 4.1].

Let $T: U_{A} \rightarrow U_{A}$ be an automorphism of $A$-algebras, and let $M$ be a representation of $U_{A}$, i.e. an $A$-algebra homomorphism $\rho_{M}: U_{A} \rightarrow \operatorname{End}_{A}(M)$. We define a $T$-twisted representation ${ }^{T} M$ by letting $U_{A}$ act on $M$ by the homomorphism ${ }^{T} \rho_{M}=\rho_{M} \circ T$. If $T\left(U_{A}^{0}\right) \subseteq U_{A}^{0}$ we can twist $U_{A}^{0}$ representations in the same way.

Lemma 2. Let $T: U_{A} \rightarrow U_{A}$ be an A-algebra endomorphism with $T\left(U_{A}^{0}\right) \subseteq$ $U_{A}^{0}$ and let $V$ be a $U_{A}^{0}$-module. Then $T$ induces a homomorphism of $U_{A}$ modules

$$
\phi:{ }^{T} \operatorname{Hom}_{U_{A}^{0}}\left(U_{A}, V\right) \longrightarrow \operatorname{Hom}_{U_{A}^{0}}\left(U_{A},{ }^{T} V\right), \quad f \longmapsto f \circ T
$$

(Recall that the untwisted $U_{A}$-module structure is given by (5).) Moreover, if $T$ is an isomorphism (of A-algebras) then $\phi$ is a module isomorphism (with inverse $\left.f \mapsto f \circ T^{-1}\right)$.

Proof. This is straightforward. 
Lemma 3. The functor $\mathscr{F}: \mathscr{U}_{A} \rightarrow \mathscr{C}_{A}$ is a left exact and commutes with direct sums.

Proof. Let $M$ be a $U_{A}$-module. First we show that $\mathscr{F}(M)$ is indeed a $U_{A^{-}}$ module: for example, if $x \in \mathscr{F}(M)$, say $E^{(s)} x=0$ for $s>s_{0}$ and $F^{(t)} x=0$ for $t>t_{0}$, then $E^{(r)} x$ and $F^{(r)} x$ are also in $\mathscr{F}(M)$ (for all $r \in \mathrm{N}$ ), for $F^{(t)} F^{(r)} x=0$ and, using Kac's formula (compare [3], 3.1.9),

$$
E^{(s+r)} F^{(r)} x=\sum_{i=0}^{r} F^{(r-i)}\left[\begin{array}{c}
K ; 2 i-2 r-s \\
i
\end{array}\right] E^{(s+r-i)} x=0
$$

(and similarly for $E^{(r)} x$ ). It is easy to see that $\mathscr{F}$ is a functor.

To show that this functor is left exact, it suffices to prove that it preserves kernels. Let $\phi: M \rightarrow N$ be a morphism of $\mathscr{U}_{A}$ :

$$
\operatorname{ker}(\mathscr{F} \phi)=\operatorname{ker}(\phi \mid \mathscr{F} M)=\operatorname{ker} \phi \cap \mathscr{F} M=\mathscr{F}(\operatorname{ker} \phi)
$$

It is easy to see that $\mathscr{F}(M \oplus N)=\mathscr{F}(M) \oplus \mathscr{F}(N)$ for all $M, N$ in $\mathscr{U}_{A}$.

Corollary 4. The functor $H_{A}^{0}\left(U_{A} / U_{A}^{0},-\right): \mathscr{C}_{A}^{\prime} \rightarrow \mathscr{C}_{A}$ is left exact and commutes with direct sums.

In the rest of this section we shall work only with one specific automorphism $T$, namely the one given by

$$
K \longmapsto K^{-1}, \quad E \longmapsto F, \quad F \longmapsto E
$$

(using (1) one checks that this is an $A$-algebra automorphism with $T\left(U_{A}^{0}\right) \subset U_{A}^{0}$.)

COROllary 5. With $T$ as in (6), there is a $U_{A}$-isomorphism

$$
\begin{aligned}
{ }^{T} H^{0}\left(U_{A} / U_{A}^{0}, V\right) & \cong H^{0}\left(U_{A} / U_{A}^{0},{ }^{T} V\right) \\
f & \longmapsto f \circ T
\end{aligned}
$$

Proof. First, $\phi$ of Lemma 2 is an isomorphism. From the identity

$$
\left({ }^{T} M\right)_{m}={ }^{T}\left(M_{-m}\right), \quad\left(M \text { any } U_{A} \text {-module }\right)
$$

and from $T\left(E^{(r)}\right)=F^{(r)}, T\left(F^{(r)}\right)=E^{(r)}$ we deduce that ${ }^{T} \mathscr{F}(M)=\mathscr{F}\left({ }^{T} M\right)$; with this identification $\mathscr{F} \phi$ is the required isomorphism.

One may check that $T$-twist (with $T$ given by (6)) is an equivalence functor from $\mathscr{U}_{A}$ to itself (In particular, the functor is faithfully exact.) The restriction of this functor maps $\mathscr{C}_{A}$ to itself.

Lemma 6. If $m \in \mathbf{Z}, V \in \mathscr{C}_{A}^{0}$ and $V_{n}=0$ for $n<-m$, then there is an isomorphism 


$$
\begin{aligned}
& H_{A}^{0}\left(U_{A} / U_{A}^{0}, V\right)_{m} \cong \\
& \left\{\left(a_{r s}\right)_{(r, s) \in \mathrm{N} \times \mathrm{N}} \mid a_{r s} \in V_{m+2(s-r)}, a_{r s}=0 \text { for } s \gg 0 \text { and all } r\right\}
\end{aligned}
$$

$$
f \longmapsto\left(f\left(F^{(r)} E^{(s)}\right)\right)
$$

Proof. First we observe that any $f \in H_{A}^{0}\left(U_{A} / U_{A}^{0}, V\right)_{m}$ is given uniquely by its values on $F^{(r)} E^{(s)}, r, s \geq 0$ (since these constitute a basis for $U_{A}$ over $U_{A}^{0}$, see [2, 6.7]). Put $a_{r s}=f\left(F^{(r)} E^{(s)}\right)$; since $f$ has weight $m$ we get

$$
\begin{aligned}
q^{m} a_{r s} & =q^{m} f\left(F^{(r)} E^{(s)}\right)=(K . f)\left(F^{(r)} E^{(s)}\right)=f\left(F^{(r)} E^{(s)} K\right) \\
& =q^{2(r-s)} K f\left(F^{(r)} E^{(s)}\right)=q^{2(r-s)} K a_{r s}
\end{aligned}
$$

(and similarly for the other generators of $U_{A}^{0}$ ) so $a_{r s}$ has weight $m+2(s-r)$. Conversely, if $a_{r s} \in V_{m+2(s-r)}$ for all $r, s \geq 0$ then

$$
\left(u F^{(r)} E^{(s)} \mapsto u a_{r s}\right), u \in U_{A}^{0}
$$

defines a function $U_{A} \rightarrow V$ that clearly belongs to $H_{U_{A}^{0}}\left(U_{A}, V\right)_{m}$.

Consider first any $f \in H_{A}^{0}\left(U_{A} / U_{A}^{0}, V\right)_{m}$ :

$$
\begin{aligned}
& \exists s_{0}>0 \forall s_{1}>s_{0} \quad: E^{\left(s_{1}\right)} f=0 \\
\Longleftrightarrow & \exists s_{0}>0 \forall s_{1}>s_{0} \forall r, s \geq 0: f\left(F^{(r)} E^{(s)} E^{\left(s_{1}\right)}\right)=0 \\
\Longleftrightarrow & \exists s_{0}>0 \forall s_{1}>s_{0} \forall r, s \geq 0:\left[\begin{array}{c}
s+s_{1} \\
s
\end{array}\right] a_{r, s+s_{1}}=0 \\
\Longleftrightarrow & \exists s_{0}>0 \forall s_{1}>s_{0} \forall r \quad \geq 0: a_{r, s_{1}}=0
\end{aligned}
$$

This proves that $f$ is indeed sent to the RHS of (8).

Conversely, let $\left(a_{r s}\right)$ from the RHS of (8) be given, and consider the corresponding function, call it $f$, as given by (10). By (11) above we deduce that $E^{(s)} f=0$ for $s \gg 0$ and we need only show that a sufficiently high power of $F$ kills $f$ :

$$
\begin{aligned}
& \exists j_{0}>0 \forall j>j_{0} \quad: F^{(j)} \cdot f=0 \\
\Longleftrightarrow & \exists j_{0}>0 \forall j>j_{0} \forall r, s \geq 0:\left(F^{(j)} \cdot f\right)\left(F^{(r)} E^{(s)}\right)=0 \\
\Longleftrightarrow & \exists j_{0}>0 \forall j>j_{0} \forall r, s \geq 0: f\left(F^{(r)} E^{(s)} F^{(j)}\right)=0 \\
\Longleftrightarrow & \exists j_{0}>0 \forall j>j_{0} \forall r, s \geq 0: \\
& f\left(\sum_{t=0}^{\min \{j, s\}}\left[\begin{array}{c}
r+j-t \\
r
\end{array}\right] F^{(r+j-t)}\left[\begin{array}{c}
K ; 2 t-j-s \\
t
\end{array}\right] E^{(s-t)}\right)=0
\end{aligned}
$$




$$
\begin{aligned}
\Longleftrightarrow & \exists j_{0}>0 \forall j>j_{0} \forall r, s \geq 0: \\
& \sum_{t=0}^{\min \{j, s\}}\left[\begin{array}{c}
r+j-t \\
r
\end{array}\right]\left[\begin{array}{c}
K ; 2 r+j-s \\
t
\end{array}\right] f\left(F^{(r+j-t)} E^{(s-t)}\right)=0 \\
\Longleftrightarrow & \exists j_{0}>0 \forall j>j_{0} \forall r, s \geq 0: \\
& \sum_{t=0}^{\min \{j, s\}}\left[\begin{array}{c}
r+j-t \\
r
\end{array}\right]\left[\begin{array}{c}
m+s-j \\
t
\end{array}\right] a_{r+j-t, s-t}=0
\end{aligned}
$$

Note that for $r-s>m$ we get $m+2(s-r)<-m$ and hence $a_{r s}=0$ by the assumption that $\mathrm{V}$ has no weights below $-m$. We shall prove (12) by considering two cases:

$m+s-j<0:(r+j-t)-(s-t)=r+j-s>r+m \geq m$, so $a_{r+j-s, s-t}=$ 0 for all $t$.

$m+s-j \geq 0$ : In this case $\left[\begin{array}{c}m+s-j \\ t\end{array}\right]=0$ for $t>m+s-j$; and if $0 \leq t \leq m+s-j$ we have $s-t \geq j-m$, whence it follows that $a_{r+j-t, s-t}=0$ (according to (12)) if we choose $j_{0}$ greater than $m+s_{0}$ (and greater than 0 ), which we may do without loss of generality.

LEMMA 7. If $m \in \mathrm{Z}$ and

$$
0 \longrightarrow P \longrightarrow Q \stackrel{\pi}{\longrightarrow} R \longrightarrow 0
$$

is an exact sequence in $\mathscr{C}_{A}^{\prime}$ and $P_{n}=Q_{n}=R_{n}=0$ for $n<-m$ then there is an exact sequence of $U_{A}^{0}$-modules

$$
0 \rightarrow H_{A}^{0}\left(U_{A} / U_{A}^{0}, P\right)_{m} \rightarrow H_{A}^{0}\left(U_{A} / U_{A}^{0}, Q\right)_{m} \stackrel{\tilde{\pi}}{\rightarrow} H_{A}^{0}\left(U_{A} / U_{A}^{0}, R\right)_{m} \rightarrow 0
$$

Proof. According to Corollary 4 we only have to prove that $\tilde{\pi}$ is surjective. Choose an arbitrary $g \in H_{A}^{0}\left(U_{A} / U_{A}^{0}, R\right)_{m}$ and let $b_{r s}=g\left(F^{(r)} E^{(s)}\right) \in$ $R_{m+2(s-r)}, r, s \geq 0$. For all $r, s \geq 0$ find $a_{r s} \in Q_{m+2(s-r)}$ such that $\pi\left(a_{r s}\right)=b_{r s}$ and $\quad b_{r s}=0 \Rightarrow a_{r s}=0 \quad(\pi$ is surjective). As in (10) above, let $f \in \operatorname{Hom}_{U_{A}^{0}}\left(U_{A}, V\right)_{m}$ be given by $u F^{(r)} E^{(s)} \mapsto a_{r s}\left(u \in U_{A}^{0}\right)$. By Lemma 6, $f \in H_{A}^{0}\left(U_{A}^{A} / U_{A}^{0}, Q\right)_{m}$ and clearly $\tilde{\pi}(f)=g$.

Theorem 8. The functor $H_{A}^{0}\left(U_{A} / U_{A}^{0},-\right): \mathscr{C}_{A}^{\prime} \rightarrow \mathscr{C}_{A}$ is exact.

Proof. Since $H_{A}^{0}\left(U_{A} / U_{A}^{0}, V\right)=\bigoplus_{m} H_{A}^{0}\left(U_{A} / U_{A}^{0}, V\right)_{m}$, it will suffice to prove the exactness of each $H_{A}^{0}\left(U_{A} / U_{A}^{0},-\right)_{m}$ (as a functor from $\mathscr{C}_{A}^{\prime}$ to the category of $A$-modules.) So let an arbitrary fixed $m \in \mathbf{Z}$ be given. For any $V$ in $\mathscr{C}_{A}^{\prime}$ we define $V^{\prime}=\bigoplus_{n \geq-m} V_{n}$ and $V^{\prime \prime}=\bigoplus_{n<-m} V_{n}$; clearly $V=V^{\prime} \oplus V^{\prime \prime}$. Given a short exact sequence in $\mathscr{C}_{A}^{\prime}$

$$
0 \longrightarrow P \longrightarrow Q \longrightarrow R \longrightarrow 0
$$


we obtain two short exact sequences (of $U_{A}^{0}$-modules)

$$
\begin{gathered}
0 \longrightarrow P^{\prime} \longrightarrow Q^{\prime} \longrightarrow R^{\prime} \longrightarrow 0 \\
0 \longrightarrow P^{\prime \prime} \longrightarrow Q^{\prime \prime} \longrightarrow R^{\prime \prime} \longrightarrow 0
\end{gathered}
$$

Using Lemma 7 we find an exact sequence

$$
0 \rightarrow H_{A}^{0}\left(U_{A} / U_{A}^{0}, P^{\prime}\right)_{m} \rightarrow H_{A}^{0}\left(U_{A} / U_{A}^{0}, Q^{\prime}\right)_{m} \rightarrow H_{A}^{0}\left(U_{A} / U_{A}^{0}, R^{\prime}\right)_{m} \rightarrow 0
$$

and by the exactness of the $T$-functor an exact sequence

$$
0 \longrightarrow{ }^{T} P^{\prime \prime} \longrightarrow{ }^{T} Q^{\prime \prime} \longrightarrow{ }^{T} R^{\prime \prime} \longrightarrow 0
$$

Since $\left({ }^{T} P^{\prime \prime}\right)_{n}={ }^{T}\left(P_{-n}^{\prime \prime}\right)=0$ for $-n \geq-m$, i.e. for $n \leq m$, we can apply Lemma 7 again to obtain an exact sequence

$$
\begin{aligned}
0 & \rightarrow H_{A}^{0}\left(U_{A} / U_{A}^{0},{ }^{T} P^{\prime \prime}\right)_{m} \rightarrow H_{A}^{0}\left(U_{A} / U_{A}^{0},{ }^{T} Q^{\prime \prime}\right)_{m} \\
& \rightarrow H_{A}^{0}\left(U_{A} / U_{A}^{0},{ }^{T} R^{\prime \prime}\right)_{m} \rightarrow 0
\end{aligned}
$$

Using Corollary 5 and (7) we deduce that the sequence

$$
\begin{aligned}
0 & \rightarrow{ }^{T}\left(H_{A}^{0}\left(U_{A} / U_{A}^{0}, P^{\prime \prime}\right)_{m}\right) \rightarrow{ }^{T}\left(H_{A}^{0}\left(U_{A} / U_{A}^{0}, Q^{\prime \prime}\right)_{m}\right) \\
& \rightarrow{ }^{T}\left(H_{A}^{0}\left(U_{A} / U_{A}^{0}, R^{\prime \prime}\right)_{m}\right) \rightarrow 0
\end{aligned}
$$

is exact, and, since $T$ is faithfully exact, that

$$
\begin{aligned}
0 & \longrightarrow H_{A}^{0}\left(U_{A} / U_{A}^{0}, P^{\prime \prime}\right)_{m} \longrightarrow H_{A}^{0}\left(U_{A} / U_{A}^{0}, Q^{\prime \prime}\right)_{m} \\
& \longrightarrow H_{A}^{0}\left(U_{A} / U_{A}^{0}, R^{\prime \prime}\right)_{m} \longrightarrow 0
\end{aligned}
$$

is exact. Finally, applying Corollary 4 to (13) and (14) yields an exact sequence

$$
0 \longrightarrow H_{A}^{0}\left(U_{A} / U_{A}^{0}, P\right)_{m} \longrightarrow H_{A}^{0}\left(U_{A} / U_{A}^{0}, Q\right)_{m} \longrightarrow H_{A}^{0}\left(U_{A} / U_{A}^{0}, R\right)_{m} \longrightarrow 0
$$

as desired.

\section{Applications}

We can define an induction functor

$$
H^{0}\left(U_{A} / U_{A}^{-} U_{A}^{0},-\right)=\mathscr{F} \circ \operatorname{Hom}_{U_{A}^{-} U_{A}^{\prime}}\left(U_{A},-\right)
$$

from the category of integrable $U_{A}^{-} U_{A}^{0}$-modules to $\mathscr{C}_{A}$. This functor is left exact but not exact, so we let $H^{i}\left(U_{A} / U_{A}^{-} U_{A}^{0},-\right)$ denote the $i$ th derived functor (the category of integrable $U_{A}^{-} U_{A}^{0}$-modules has enough injectives). This functor is often written quite simply as $H_{A}^{0}(-)$ and the derived functors as 
$H_{A}^{i}(-)$. As in [4, section 2] we may use Theorem 8 to prove vanishing theorems. We may extend the $U_{A}^{0}$-module $\chi_{m}$ to a $U_{A}^{-} U_{A}^{0}$-module by letting $U_{A}^{-}$ act trivially. Then we have:

Proposition 9 (Kempf vanishing). Let $m \geq 0$. Then $H_{A}^{i}\left(\chi_{m}\right)=0$ for $i>0$.

Proof. [4, 2.4]

Proposition 10. $H^{i}(-)=0$ for $i>1$

Proof. $[4,2.5]$ or $[1,4.3]$

Let $k$ be a field where we choose a distinguished element $\xi \in k^{\times}$; we may then consider $k$ as an $A$-algebra by $q \mapsto \xi$. We have then a quantum algebra $U_{k}=k \otimes_{A} U_{A}$ with a decomposition as (2), $U_{k}=U_{k}^{-} U_{k}^{0} U_{k}^{+}$, where $U_{k}^{0}=k \otimes_{A} U_{A}^{0}$ and similarly for $U_{k}^{-}$and $U_{k}^{+}$. We now consider $E^{(r)}, F^{(r)}, K, K^{-1}$ and $\left[\begin{array}{c}K ; c \\ t\end{array}\right]$ as elements of $U_{k}$. We may then define for $m \in \mathbf{Z}$ characters $\chi_{m}: U_{k} \rightarrow k$ by composing the map in (3) with the algebra map $A \rightarrow k$. We also extend the concept of integrable modules to $U_{k}$-modules (resp. $U_{k}^{0}$-modules), and we have then an induction functor as in (4) which we denote $H_{k}^{0}\left(U_{k} / U_{k}^{-} U_{k}^{0},-\right)$, or simply $H_{k}^{0}(-)$.

Proposition 11. Let $V$ be an integrable $U_{A}^{0}$-module. Then $[4,2.9]$

$$
H_{k}^{0}\left(k \otimes_{A} V\right) \cong k \otimes_{A} H_{A}^{0}(V)
$$

Proof. As in the proof of Theorem 8 we write $V=V^{\prime} \oplus V^{\prime \prime}$ where $V^{\prime}=\bigoplus_{n \geq-m} V_{n}$ and $V^{\prime \prime}=\bigoplus_{n<-m} V_{n}$. In the same notation, $(k \otimes V)^{\prime}=k \otimes\left(V^{\prime}\right)$ and $(k \otimes V)^{\prime \prime}=k \otimes\left(V^{\prime \prime}\right)$ since $(k \otimes V)_{n}=k \otimes V_{n}$. Using Lemma 6 and a similar version for $H^{0}\left(U_{k} / U_{k}^{0},-\right)$, we see that

$$
\begin{aligned}
k \otimes H^{0}\left(U_{A} / U_{A}^{0}, V^{\prime}\right)_{m} & =k \otimes\left\{\left(a_{r s}\right) \mid a_{r s} \in V_{m+2(s-r)}, a_{r s}=0, s \gg 0\right\} \\
& =\left\{\left(\bar{a}_{r s}\right) \mid \bar{a}_{r s} \in\left(k \otimes V^{\prime}\right)_{m+2(s-r)}, \bar{a}_{r s}=0, s \gg 0\right\} \\
& =H^{0}\left(U_{k} / U_{k}^{0}, k \otimes V^{\prime}\right)_{m}
\end{aligned}
$$

As in Corollary 5, we have for each $U_{k}^{0}$-module $M$ an isomorphism of $U_{k^{-}}$ modules

$$
{ }^{T} H^{0}\left(U_{k} / U_{k}^{0}, M\right) \cong H^{0}\left(U_{k} / U_{k}^{0},{ }^{T} M\right)
$$

and then, using Corollary 5, (16), and (15) with ${ }^{T} V^{\prime \prime}$ and $-m$ substituted for, respectively, $V^{\prime}$ and $m$, 


$$
\begin{aligned}
& T_{\left(\left(k \otimes H^{0}\left(U_{A} / U_{A}^{0}, V^{\prime \prime}\right)\right)_{m}\right)} \cong k \otimes{ }^{T}\left(H^{0}\left(U_{A} / U_{A}^{0}, V^{\prime \prime}\right)_{m}\right) \\
&=k \otimes H^{0}\left(U_{A} / U_{A}^{0},{ }^{T} V^{\prime \prime}\right)_{-m} \\
& \cong H^{0}\left(U_{k} / U_{k}^{0}, k \otimes{ }^{T} V^{\prime \prime}\right)_{-m} \\
&=H^{0}\left(U_{k} / U_{k}^{0},{ }^{T}\left(k \otimes V^{\prime \prime}\right)\right)_{-m} \\
& \cong{ }^{T}\left(H^{0}\left(U_{k} / U_{k}^{0}, k \otimes V^{\prime \prime}\right)_{m}\right)
\end{aligned}
$$

whence we get

$$
H^{0}\left(U_{k} / U_{k}^{0}, k \otimes V^{\prime \prime}\right)_{m} \cong k \otimes H^{0}\left(U_{A} / U_{A}^{0}, V^{\prime \prime}\right)_{m}
$$

Finally, we take the direct sum of (15) and (17) and use that also $H^{0}\left(U_{k} / U_{k}^{0},-\right)$ commutes with direct sums (cf. Corollary 4).

\section{REFERENCES}

1. Henning Haahr Andersen, Patrick Polo, and Wen Kexin, Representations of quantum algebras, Invent. Math. 104 (1991).

2. George Lusztig, Quantum Groups at roots of 1, Geom. Dedicata 35 (1990).

3. George Lusztig, Introduction to quantum groups, Progr. Math. 110 (1993).

4. Lars Thams, Two classical results in the quantum mixed case, J. Reine Angew. Math. 436 (1993).

INSTITUTE OF MATHEMATICS

UNIVERSITY OF AARHUS

NY MUNKEGADE

DK-8000 AARHUS C

DENMARK

Email: jens@mi.aau.dk 